\title{
A perspective on the applications of furin inhibitors for the treatment of SARS-CoV-2
}

\author{
Kasi Pandima Devi ${ }^{1}\left[\right.$ Mahmoud Reza Pourkarim ${ }^{2,3}$ - Marijn Thijssen ${ }^{2} \cdot$ Antoni Sureda $^{4,5}$. \\ Maryam Khayatkashani ${ }^{6}$. Cosmin Andrei Cismaru ${ }^{7,8}$. Ioana Berindan Neagoe ${ }^{7,9,10}$. Solomon Habtemariam ${ }^{11}$. \\ Soha Razmjouei ${ }^{12} \cdot$ Hamid Reza Khayat Kashani ${ }^{13}$
}

Received: 2 July 2021 / Revised: 20 November 2021 / Accepted: 23 November 2021 / Published online: 15 January 2022

(c) The Author(s) under exclusive licence to Maj Institute of Pharmacology Polish Academy of Sciences 2021

\begin{abstract}
Currently, the world is facing a pandemic of the new coronavirus SARS-CoV-2 that causes COVID-19. Identifying key targets in the viral infection lifecycle is urgently needed for designing therapeutic strategies to combat the virus. Furin is a subtilisin-like proprotein convertase with diverse cellular functions. Emerging evidence suggests that furin plays a critical role in the activation and/or infectivity of SARS-CoV-2. In this perspective, we discuss the potential role of furin in the entry SARS-CoV-2 into host cells. Furthermore, we evaluate available peptide and non-peptide furin inhibitors and potential outcomes, including immune responses.
\end{abstract}

Keywords Furin inhibitor $\cdot$ SARS-CoV-2 $\cdot$ Spike protein $\cdot$ Peptide $\cdot$ Non-peptide $\cdot$ Immune responses

Kasi Pandima Devi

pdevik@alagappauniversity.ac.in; devikasi@yahoo.com

$\triangle$ Mahmoud Reza Pourkarim

mahmoudreza.pourkarim@kuleuven.be

$\triangle$ Hamid Reza Khayat Kashani

khayatkashani@sbmu.ac.ir

1 Department of Biotechnology, Alagappa University (Science Campus), Karaikudi 630 003, Tamil Nadu, India

2 Division of Clinical and Epidemiological Virology, Department of Microbiology, Immunology and Transplantation, KU Leuven, 3000 Leuven, Belgium

3 Health Policy Research Center, Institute of Health, Shiraz University of Medical Sciences, Shiraz, Iran

4 Research Group in Community Nutrition and Oxidative Stress, University of the Balearic Islands-IUNICS, Health Research Institute of Balearic Islands (IdISBa), Palma de Mallorca, Spain

5 CIBER Fisiopatología de la Obesidad y Nutrición (CIBEROBN), Instituto de Salud Carlos III (ISCIII), Madrid, Spain

6 Amol University of Special Modern Technologies, Amol, Iran
7 Research Center for Functional Genomics, Biomedicine and Translational Medicine, "Iuliu Hatieganu" University of Medicine and Pharmacy, Cluj-Napoca, Romania

8 Department of Functional Sciences, Immunology and Allergology, "Iuliu Hatieganu" University of Medicine and Pharmacy Cluj-Napoca, Cluj-Napoca, Romania

9 The Center for Advanced Medicine, Medfuture-“Iuliu Hatieganu" University of Medicine and Pharmacy, Cluj-Napoca, Romania

10 The Department for Functional Genomics and Experimental Pathology, The Oncology Institute "Prof. Dr. Ion Chiricuta", Cluj-Napoca, Romania

11 Pharmacognosy Research Laboratories and Herbal Analysis Services UK, University of Greenwich, Central Avenue, Chatham-Maritime, Kent ME4 4TB, UK

12 Department of Internal Medicine, Semnan University of Medical Sciences and Health Services, Semnan, Iran

13 Department of Neurosurgery, Imam Hossein Hospital, Shahid Beheshti University of Medical Sciences, 1617763141 Tehran, Iran 


\section{Introduction}

The world is currently facing a devastating pandemic caused by the severe acute respiratory syndrome coronavirus-2 (SARS-CoV-2, previously known as 2019-nCoV) [1]. Patients infected with this virus display a variety of symptoms that are collectively referred to as coronavirus disease-2019 (COVID-19). The disease originated in Wuhan, Hubei province, China in December 2019, and quickly spread on globally to reach $235,673,032$ confirmed cases and 4,814,651 deaths at 3 October, 2021. Currently, more than 213 countries are affected and the peak of the infection curve is still out of sight [2]. Genomic characterization of the virus was carried out by scientists from China following isolation of the virus from throat swabs of hospitalized patients in Wuhan. The initial patients were linked to the Huanan seafood market, which is suggested to be the origin of the new virus. Full-genome analysis of the collected strains of SARS-CoV-2 revealed a sequence identity of 87.99 and $87.23 \%$ with its close relatives batSL-CoVZC45 and bat-SL-CoVZXC21 respectively [3]. Phylogenetic analysis also revealed a close relationship of SARS-CoV-2 with the bat BatCoV-RaTG13 strain isolated in Yunnan, China and pangolin-derived viruses [4].

Coronavirus virions have a spherical structure of about $125 \mathrm{~nm}$ in diameter and are made up of four main structural proteins: the spike (S), membrane (M), envelope (E), and nucleocapsid $(\mathrm{N})$ proteins [5]. So far, infectivity studies conducted on SARS-CoV-2 have shown the critical role of angiotensin-converting enzyme II (ACE2) as a receptor for viral entry into host cells. This mechanism has also been observed in SARS-CoV and HCoV-NL63 infections. In contrast, other human coronaviruses generally use APN (aminopeptidase N), for example HCoV-229E (human coronavirus 229E), and DPP4 (dipeptidyl peptidase 4), in the case of hCoV-EMC (human coronavirus-Erasmus Medical Center), as entry receptors $[3,6,7]$. During infection, the $\mathrm{S}$ protein on the viral surface is cleaved into subunits $\mathrm{S} 1$ and S2. Subunit S1 contains the receptor binding domain that binds ACE2 on the host cells [8]. This proteolytic cleavage of the glycoproteins present on the viral envelope is required for virus cell entry [9]. Subtilisin-like endoproteases (serine proteases) or trypsin endoproteases are potential enzymes that could be used by the viruses to cleave the protein [10]. Furins/proprotein convertase subtilisin/kexin proteins are calcium $\left(\mathrm{Ca}^{2+}\right)$-dependent serine endoproteases widely present in humans. Furin (Paired Basic Amino Acid Cleaving Enzyme (PACE)) is a subtilisin-like proprotein convertases (PC) that cleaves at the Arg-X-Arg/Lys-Arg $\downarrow-X$ ( $\downarrow$ shows the cleavage site and $X$ can be any amino acid), a multi-basic cleavage motif present in the $\mathrm{S}$ protein. This cleavage by furin converts the inactive $\mathrm{S}$ protein precursors of the zoonotic Middle East respiratory syndrome coronavirus (MERS$\mathrm{CoV}$ ) into active proteins that induce infection $[11,12]$. Furin is expressed in the trans-Golgi network and the cell membrane, mediated by endosomal translocation [13]. Although, furin is considered a transmembrane protein, it can also be released as an active enzyme into the extracellular space after cleavage [14].

A recent report reveals that the SARS-CoV-2 contains a cleavage site of four aminoacids (PRRA) at positions 681-684 between the S1 and S2 subunits of the S glycoprotein. Although the cleavage site is not completely identical, a similar insertion has been shown for MERS-CoV and several other coronaviruses [12]. This insert contains a functional furin cleavage motif PRRARSV at the S1/S2 site, suggesting that cleavage by furin is indispensable for SARS-CoV-2 entry into host cells [15]. However, S protein activation is a complex process and involves more than one cleavage site (S1/S2 and S2') as well as distinct host cell proteases (trypsin-like proteases, cathepsins and furin). A recent study has shown that SARS-CoV-2 entry is also dependent on transmembrane protease, serine 2 (TMPRSS2) and cathepsins [16]. Moreover, while furin has been reported to promote SARS-CoV-2 infectivity and cell spread, it is not essential as it was evidenced using CRISPR-Cas9 knockout cell line [17]. The results evidenced that $\mathrm{S}$ protein processing can occur independent of furin although the presence of the protease significantly increases cleavage. The authors conclude that furin inhibitors may reduce but not completely abolish viral spread. Anyway, considering the key role played by the $\mathrm{PC}$ furin in the pathogenicity and infectivity of many viruses including the SARS-CoV-2, inhibitory molecules of furin have enormous therapeutic potential and could be a potential drug target to combat COVID-19[18]. Hence, a potent furin inhibitor could offer a broad-spectrum antiviral effect against SARS-CoV-2 and related viruses that depend on furin for their infectivity (Fig. 1). Here, we provide an overview of furin inhibitors identified that could

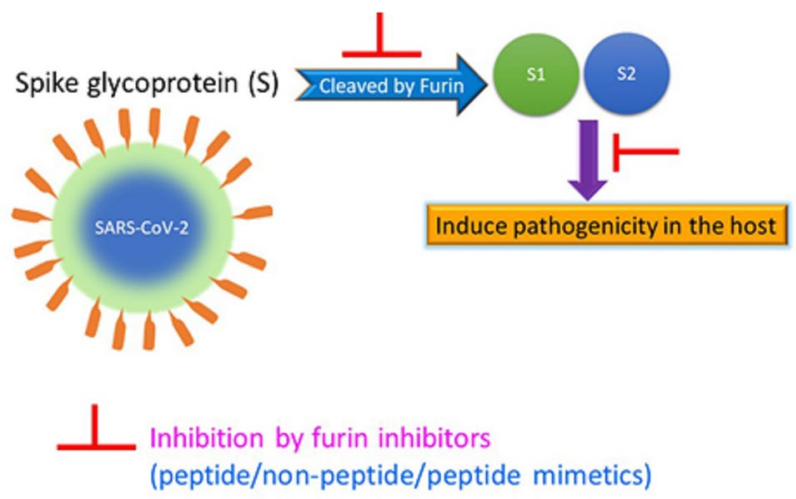

Fig. 1 Furin inhibitors as therapeutic target for SARS-CoV-2 
be tested for their efficacy against SARS-CoV-2. However, an important aspect to consider is the possible existence of mutations in protein $\mathrm{S}$, which may affect the $\mathrm{S} 1 / \mathrm{S} 2$ cleavage site, and confer resistance to higher susceptibility to furininduced cleavage and, thus altering viral entry and cell-cell fusion [19]. In this sense, it has evidenced that mutations at the S2' site of SARSCoV-2 S significantly reduce S1/S2 border cleavage leading to altered furin loop structure and rendering it inaccessible for the enzyme [20]. In a study using mutant SARS-CoV-2 that lacks the furin cleavage site it was observed a decrease in the replication of the virus in a human respiratory cell line and attenuated its ability to cause disease in vivo. Furthermore, infection with the mutant conferred protection against re-exposure to parental SARS-CoV-2 [21]. In addition, some vaccines in development such as Biovacc-19 are aimed at developing antibodies against those parts of the peak protein of SARS-CoV-2 that participate in the binding and infection of cells, such as the cleavage sites for furin [22].

In humans, furin is involved in enzymatic processing of precursor proteins into their mature forms. The PC family members, including furin, exert their function on proteins which are vital for normal physiological processes. These include plasma proteins, hormones, growth factors, receptors, and matrix metalloproteases. Accordingly, knockdown of furin coding gene during embryonic development has lethal consequences [23]. Furins are also involved in many pathological processes. Accumulating evidence suggests that targeting furins could represent therapeutic strategies against bacterial toxins, viral pathogens [23, 24], and malignant diseases [25]. However, the presence of furin but also ACE2 and TMPRSS2 gene variants could modulate viral infectivity amongst humans, making some people less or more vulnerable than others to Covid-19. For example, some deleterious variants found in furin which are frequent in the Middle Eastern but not in the European populations may confer possible protective effects against the SARSCoV-2 [26].

\section{The role of furins in immune responses}

Furins, as well as other members of the PC family, serve as pro-TGF $\beta-1$ (Transforming growth factor beta) converting enzymes [27]. This has been demonstrated by an impaired TGF $\beta-1$ production after furin deletion in T cells. Therefore, furin is considered as an important mediator of regulatory $\mathrm{T}$ cell (Treg) activity via TGF $\beta-1$ signaling. Furthermore, furin has been linked to the secretion of interferon- $\gamma$ (IFN- $\gamma$ ) by $\mathrm{T}$ helper type 1 (Th1) cells, an important cytokine that confers protection against numerous intracellular pathogens [28]. The expression of furin depends on the activation of signal transducer and activator of transcription 4 (STAT4), regulated by interleukin (IL)-12 signaling [29]. The crucial role of furin in regulating the immune response is further supported by a decreased IL-2 expression, unaltered levels of tumor necrosis factor (TNF) or IL-17 and higher expression of Th1 (IFN- $\gamma$ ) and Th2 (IL-4 and IL-13) cytokines in furin deficient $\mathrm{T}$ cells. Moreover, inhibition of furin resulted in a breakdown of peripheral immune tolerance demonstrated by the presence of autoantibodies and excessive production of IFN- $\gamma$, IL-4 and IL-13 in mice. Selective deletion of furin in $\mathrm{T}$ cells resulted in an autoimmunity phenotype through dysregulated Treg function [30]. The immune-activating characteristics of furin inhibition might be beneficial in boosting $\mathrm{T}$ cell-mediated immune responses in pathogenic infections and cancer [31]. However, targeting furin might result in a disruption of peripheral tolerance and stimulate autoimmune reactions [31].

\section{Furin inhibition and SARS-CoV-2 infection}

The presence of a furin cleavage site between the S1/S2 subunits suggests that SARS-CoV-2 relies on proteolytic cleavage to improve viral infectivity. This hypothesis implies that the activity of furin is paramount to the virus infection cycle and could, therefore, be an attractive target to reduce the virulence of SARS-CoV-2. The effect of furin inhibition might suppress infection by two-fold via restricting viral entry into host cells and enhancing the immune response to promote viral clearance. Several pro-toxins and membrane fusion proteins of multiple viruses and bacteria, including Ebola virus, Marburg virus, bird flu virus (A H5N1), and toxins of anthrax and botulinum, exploit host cell surface PCs processing for their entry/infection [24, 32]. Hence, using furin antagonists may be used as prophylaxis against multiple furin-dependent pathogens [24]

Several compounds have shown to possess furininhibitory potency. For instance, D-Arg-based peptides, $\alpha 1$-antitrypsin Portland (used in HIV Env inhibition [33]), and decanoyl-Arg-Val-Lys-Arg-chloromethylketone (decRVKR-Cmk) [34] have been used in vitro to inhibit furin and other PCs in the past. Currently, available furin inhibitors are non-peptide compounds, pure peptides, or peptide mimetics. Peptides have various limitations such as their degradation or limited absorption in the intestinal tract or the fact that due to proteases, agglutination and opsonization, free peptides are not systemically stable without additional modifications [35]. For example, the synthetic polyarginine cationic and cell-penetrating hexa-D-arginine amide and nona-D-arginine amide are peptide based protease inhibitors that inhibit both furin and other PCs [36]. These peptides were further modified by macrocyclization to improve the compound stability. To date, several macrocyclic peptidomimetic drugs have been synthesized and have demonstrated the capability to inhibit furin by binding its active site. However, these 
cyclic peptides did not affect viral replication in cell culturebased assays [37].

The peptidyl derivative dec-RVKR-cmk, a peptidomimetic molecule synthesized from chloromethyl ketone, is a furin convertase inhibitor. Such molecules inhibit PCs by binding the catalytic site and are called substrate homologous/analogue inhibitors, which restricts the replication of viruses that rely on furin for infecting host cells [34]. Chemical modification of these compounds has substantially improved the furin inhibiting potential. For example, C-terminal modification of dec-RVKR-cmk with decarboxylated arginine mimetics resulted in highly potent furin inhibitors [38]. The presence of an Arg residue at P1, the residue N-terminal to the scissile peptide bond (Fig. 1), and the furin cleavage, was the rationale for synthesizing substrate analogues with decarboxylated arginine mimetics. The P1 residue of these furin inhibitors, such as the phenylacetylArg-Val-Arg-4-amidinobenzylamide, can form hydrogen bonds with the $\mathrm{S} 1$ binding pocket of furin and results in a better binding affinity [39]. Recently, the synthetic peptide mimetic MI-1851 has been shown to inhibit furin at $10 \mu \mathrm{M}$ in the human lung cancer Calu-3 cells and prevented the spread of the SARS-CoV-2. The virus titer was reduced up to 75-fold at this concentration. In addition, when MI-1851 was combined with T-ex5 PPMO (an inhibitor of transmembrane serine protease 2; TMPRSS2), complete blockade in the replication of SARS-CoV-2 was observed [40]. This suggests that the combination therapies which prevent the viral activation pathway would be more effective in the management of SARS-CoV-2.

The specificity of furin relies on the amino acid side chains present in the cleavage site. For instance, furin requires an Arg residue in P1 and positions P2, P4 and P6 must contain at least two residues of either Arg or Lys. Furthermore, no aliphatic or hydrophobic amino acid should be present at the P1' site. These concepts were used to design protein-based inhibitors [41]. For example, engineered proteins like the $\alpha 1-\mathrm{PDX} / \mathrm{hf}(\alpha 1-\mathrm{AT}$ Portland/His and FLAGtagged, an engineered product of the Alpha-1 antitrypsin) which are similar to SERPINs (serine protease inhibitors), act as protease inhibitors. A kinetics study revealed that $\alpha 1-P D X$ inhibits furin by establishing tight interactions after the formation of an initial loose complex. Generally, inhibiting proteins engineered to be selective for furin, contain the specific cleavage site -ArgP4-Xaa-Xaa-Arg-P1 [42].

In addition to proteins and peptides, small moleculebased competitive furin inhibitors have been developed. The benefit of these drugs is that they do not require proteolytic degradation and that these compounds are stable and accessible. Since the active site of furin is negatively charged, positively charged inhibitors are frequently used as furin inhibitors, like Guanidinylated Aryl 2,5-Dideoxystreptamine Derivatives (GADDs). A study demonstrated the anti-furin activity of GADDs at nanomolar concentration range, which makes these molecules interesting candidates for antiviral applications [43]. To better understand the mode of action of DDs, structural studies were performed with different synthesized inhibitors. One 2,5-dideoxystreptamine derived inhibitor was reported to affect the function of furin by binding to the $\mathrm{S} 4$ pocket forming a charged hydrogen bond with Asp153 and interfering with conformation and function of the catalytic site while another derivate interacted with a planar peptide stretch that includes Asp228-Glu230 which is a less conserved region [34]. This study indicates that the binding of the derivatives to non-conserved regions could be the basis for developing new inhibitors. Of note, these compounds are not required to be positively charged to establish electrostatic interaction with the active site of furin, which overcomes the limitation of poor cell permeability [44]. In another study, guanyl hydrazone inhibitors evidenced an active site-directed binding mode to the furin OFF-state conformation [45]. The compounds were found to interact with the $\mathrm{S} 1$ pocket and with a second binding site at the S4 / S5 pocket of furin.

Besides synthetic compounds, natural products have also shown antagonistic properties against furin. For example, small molecule PC inhibitors were isolated from the herbaceous plant Andrographis paniculate and named succinoyl esters of andrographolide (SEA). These compounds are based on andrographolide and neoandrographolide skeletons and inhibit furin in a micromolar concentration range. Their bioactivity originates from the presence of andrographolide14-deoxy-3,19-O-disuccinate [46]. Furthermore, the flavonoid baicalein was demonstrated to exert anti-proliferative activity mediated through the inhibition of furin [47]. Finally, tetrahydroxy flavone luteolin exhibited uncompetitive inhibition of human furin in in vitro studies which suggests that this compound could limit viral infectivity [48].

The ubiquitous expression of furin-like enzymes and the involvement in a multitude of cellular processes, including immune reactivity and pathogenesis of malignant disease, should be considered when applying furin inhibitors to avoid systemic inhibition and subsequent immune-related toxicity. While furin inhibitors might restrict viral entry of SARS-CoV-2 in host cells, their usage requires a comprehensive management of potential autoimmune reactions. For instance, a similar approach that has been adopted to trace the effect of immune check-point inhibitors in cancer immunotherapy could be proposed [49].

Upon inhalation of viral particles, bronchial epithelial cells are among the first cells with surface-associated PCs exposed to SARS-CoV-2. Therefore, developing an inhalation-based system of nanoparticle-immobilized drugs to optimize the delivery of small molecule furin inhibitors, and minimizing potential systemic side-effects, could offer perspectives for COVID-19 treatment. The potential use of 
this approach has been demonstrated in highly pathogenic avian influenza H5N1 HA and successfully inhibited furin and related PCs [24]. While enteral and parenteral administration of furin inhibitors may require systemic immunosuppression to prevent autoimmune reactions, an inhalation route of administering furin inhibitors might require inhaled corticosteroids to prevent or treat possible immune-related pneumonitis [50].

This article provided a comprehensive overview of the available furin inhibitors which can be used for controlling disease-related processes. Some of the molecules like the dec-RVKR-cmk have been shown to inhibit the processing of SARS-CoV-2 [9]. However, most of the molecules have not been tested for their efficacy against SARS-CoV-2, albeit based on the genomic organization of the virus; furin inhibitors could be considered as one of the therapeutic strategies for developing new drugs to combat COVID-19. In addition, other promising molecules like helicase inhibitors [51], cholesterol lowering drugs like statins [52-54] and natural products like curcumin [55] which have been recently suggested for the treatment of SARS-CoV-2, could be given as a combination therapy with furin inhibitors for more effective treatment.

Acknowledgements KPD wish to acknowledge the (i) DBT-Bioinformatics Infrastructure Facility (File No.BT/BI/25/012/2012,BIF); (ii) DST-FIST (Grant No. SR/FST/LSI-639/2015(C)); (iii) UGC-SAP (Grant No. F.5-1/2018/DRS-II (SAP-II)); (iv) DST-PURSE (Grant No. SR/PURSE Phase 2/38 (G)),Rc S.O. (P)/DST-PURSE Phase II/ P1464/2019; and (v) RUSA 2.0 [F. 24-51/2014-U, Policy (TN MultiGen), Department of Education, Govt. of India].

Author contributions KPD, MRP, MT, MKCAC and IBN wrote the manuscript and were involved with project concept and submission. $\mathrm{SH}$ and HRKK revised the manuscript and were responsible for final approval; all authors contributed to this manuscript.

Funding A. Sureda was supported by Instituto de Salud Carlos III, Spain (CIBEROBN CB12/03/30038).

\section{Declarations}

Conflict of interest The authors declare that the research was conducted in the absence of any commercial or financial relationships that could be construed as a potential conflict of interest.

\section{References}

1. CfD C. Prevention. Novel coronavirus (2019-nCoV) Situation Summary. Geneva: WHO; 2019.

2. WHO. Coronavirus disease 2019 (COVID-19): situation report, 72. Geneva: WHO; 2020.

3. Xu X-W, Wu X-X, Jiang X-G, Xu K-J, Ying L-J, Ma C-L, et al. Clinical findings in a group of patients infected with the 2019 novel coronavirus (SARS-Cov-2) outside of Wuhan, China: retrospective case series. BMJ. 2020;368:m792.
4. Lam TT-Y, Shum MH-H, Zhu H-C, Tong Y-G, Ni X-B, Liao Y-S, et al. Identifying SARS-CoV-2 related coronaviruses in Malayan pangolins. Nature. 2020;583:282-5.

5. Fehr AR, Perlman S. Coronaviruses: an overview of their replication and pathogenesis. Methods Mol Biol (Clifton). 2015;1282:1-23.

6. Raj VS, Mou H, Smits SL, Dekkers DH, Müller MA, Dijkman R, et al. Dipeptidyl peptidase 4 is a functional receptor for the emerging human coronavirus-EMC. Nature. 2013;495(7440):251-4.

7. Yeager CL, Ashmun RA, Williams RK, Cardellichio CB, Shapiro LH, Look AT, et al. Human aminopeptidase $\mathrm{N}$ is a receptor for human coronavirus 229E. Nature. 1992;357(6377):420-2.

8. Wang Q, Zhang Y, Wu L, Niu S, Song C, Zhang Z, et al. Structural and functional basis of SARS-CoV-2 entry by using human ACE2. Cell. 2020;181:894-904.

9. Hoffmann M, Kleine-Weber H, Pöhlmann S. A multibasic cleavage site in the spike protein of SARS-CoV-2 is essential for infection of human lung cells. Mol Cell. 2020;78(4):779-84.e5.

10. Coutard B, Valle C, de Lamballerie X, Canard B, Seidah N, Decroly E. The spike glycoprotein of the new coronavirus 2019$\mathrm{nCoV}$ contains a furin-like cleavage site absent in $\mathrm{CoV}$ of the same clade. Antivir Res. 2020;176:104742.

11. Qian Z, Dominguez SR, Holmes KV. Role of the spike glycoprotein of human Middle East respiratory syndrome coronavirus (MERS-CoV) in virus entry and syncytia formation. PLoS ONE. 2013. https://doi.org/10.1371/journal.pone.0076469.

12. Millet JK, Whittaker GR. Host cell entry of Middle East respiratory syndrome coronavirus after two-step, furin-mediated activation of the spike protein. Proc Natl Acad Sci. 2014;111(42):15214-9.

13. Han J, Wang Y, Wang S, Chi C. Interaction of Mint3 with Furin regulates the localization of Furin in the trans-Golgi network. J Cell Sci. 2008;121(13):2217-23.

14. Evdokimov K, Biswas S, Adrian M, Weber J, Schledzewski K, Winkler M, et al. Proteolytic cleavage of LEDA-1/PIANP by furin-like proprotein convertases precedes its plasma membrane localization. Biochem Biophys Res Commun. 2013;434(1):22-7.

15. Wo ZG. Sequence analysis indicates that 2019-ncov virus contains a putative furin cleavage site at the boundary of S1 and S2 domains of spike protein. Preprints. 2020. https://doi.org/10.3121/ osf.io/nkcrf.

16. Hoffmann M, Kleine-Weber H, Schroeder S, Krüger N, Herrler T, Erichsen S, et al. SARS-CoV-2 cell entry depends on ACE2 and TMPRSS 2 and is blocked by a clinically proven protease inhibitor. Cell. 2020;181:271-80.

17. Papa G, Mallery DL, Albecka A, Welch LG, Cattin-Ortolá J, Luptak J, et al. Furin cleavage of SARS-CoV-2 Spike promotes but is not essential for infection and cell-cell fusion. PLoS Pathog. 2021;17(1):e1009246.

18. Raghav PK, Kalyanaraman K, Kumar D. Human cell receptors: potential drug targets to combat COVID-19. Amino Acids. 2021;53(6):813-42.

19. Harvey WT, Carabelli AM, Jackson B, Gupta RK, Thomson EC, Harrison EM, et al. SARS-CoV-2 variants, spike mutations and immune escape. Nat Rev Microbiol. 2021;19(7):409-24.

20. Barrett CT, Neal HE, Edmonds K, Moncman CL, Thompson R, Branttie JM, et al. Effect of clinical isolate or cleavage site mutations in the SARS-CoV-2 spike protein on protein stability, cleavage, and cell-cell fusion. J Biol Chem. 2021;297(1):100902.

21. Johnson BA, Xie X, Bailey AL, Kalveram B, Lokugamage KG, Muruato A, et al. Loss of furin cleavage site attenuates SARSCoV-2 pathogenesis. Nature. 2021;591(7849):293-9.

22. Sørensen B, Susrud A, Dalgleish AG. Biovacc-19: a candidate vaccine for Covid-19 (SARS-CoV-2) developed from analysis of its general method of action for infectivity. QRB Discov. 2020;1:e6. 
23. Thomas G. Furin at the cutting edge: from protein traffic to embryogenesis and disease. Nat Rev Mol Cell Biol. 2002;3(10):753-66.

24. Shiryaev SA, Remacle AG, Ratnikov BI, Nelson NA, Savinov AY, Wei G, et al. Targeting host cell furin proprotein convertases as a therapeutic strategy against bacterial toxins and viral pathogens. J Biol Chem. 2007;282(29):20847-53.

25. Braun E, Sauter D. Furin-mediated protein processing in infectious diseases and cancer. Clin Transl Immunol. 2019;8(8):e1073.

26. Al-Mulla F, Mohammad A, Al Madhoun A, Haddad D, Ali H, Eaaswarkhanth $\mathrm{M}$, et al. ACE2 and FURIN variants are potential predictors of SARS-CoV-2 outcome: a time to implement precision medicine against COVID-19. Heliyon. 2021;7(2):e06133.

27. Dubois CM, Blanchette F, Laprise M-H, Leduc R, Grondin F, Seidah NG. Evidence that furin is an authentic transforming growth factor- $\beta 1$-converting enzyme. Am J Pathol. 2001;158(1):305-16.

28. Oksanen A, Aittomäki S, Jankovic D, Ortutay Z, Pulkkinen K, Hämäläinen $S$, et al. Proprotein convertase FURIN constrains Th2 differentiation and is critical for host resistance against Toxoplasma gondii. J Immunol. 2014;193(11):5470-9.

29. Pesu M, Muul L, Kanno Y, O'Shea JJ. Proprotein convertase furin is preferentially expressed in T helper 1 cells and regulates interferon gamma. Blood. 2006;108(3):983-5.

30. Pesu M, Watford WT, Wei L, Xu L, Fuss I, Strober W, et al. T-cellexpressed proprotein convertase furin is essential for maintenance of peripheral immune tolerance. Nature. 2008;455(7210):246-50.

31. Vähätupa M, Aittomäki S, Martinez Cordova Z, May U, Prince S, Uusitalo-Järvinen $\mathrm{H}$, et al. T-cell-expressed proprotein convertase FURIN inhibits DMBA/TPA-induced skin cancer development. Oncoimmunology. 2016;5(12):e1245266.

32. Remacle AG, Shiryaev SA, Oh E-S, Cieplak P, Srinivasan A, Wei G, et al. Substrate cleavage analysis of furin and related proprotein convertases A comparative study. J Biol Chem. 2008;283(30):20897-906.

33. Anderson E, Thomas L, Hayflick J, Thomas G. Inhibition of HIV-1 gp160-dependent membrane fusion by a furin-directed alpha 1-antitrypsin variant. J Biol Chem. 1993;268(33):24887-91.

34. Garten W, Hallenberger S, Ortmann D, Schäfer W, Vey M, Angliker $\mathrm{H}$, et al. Processing of viral glycoproteins by the subtilisinlike endoprotease furin and its inhibition by specific peptidylchloroalkylketones. Biochimie. 1994;76(3-4):217-25.

35. Bruno BJ, Miller GD, Lim CS. Basics and recent advances in peptide and protein drug delivery. Ther Deliv. 2013;4(11):1443-67.

36. Ramos-Molina B, Lick AN, Shirazi AN, Oh D, Tiwari R, ElSayed NS, et al. Cationic cell-penetrating peptides are potent furin inhibitors. PLoS ONE. 2015. https://doi.org/10.1371/journ al.pone.0130417.

37. Lam V, van T, Ivanova $T$, Hardes $K$, Heindl MR, Morty RE, Böttcher-Friebertshäuser E, et al. Design, synthesis, and characterization of macrocyclic inhibitors of the proprotein convertase furin. ChemMedChem. 2019;14(6):673-85.

38. Imran M, Saleemi MK, Chen Z, Wang X, Zhou D, Li Y, et al. Decanoyl-Arg-Val-Lys-Arg-Chloromethylketone: an antiviral compound that acts against flaviviruses through the inhibition of furin-mediated prM cleavage. Viruses. 2019;11(11):1011.

39. Becker GL, Sielaff F, Than ME, Lindberg I, Routhier S, Day R, et al. Potent inhibitors of furin and furin-like proprotein convertases containing decarboxylated P1 arginine mimetics. J Med Chem. 2010;53(3):1067-75.

40. Bestle D, Heindl MR, Limburg H, Lam V, van T, Pilgram O, Moulton $\mathrm{H}$, et al. TMPRSS2 and furin are both essential for proteolytic activation of SARS-CoV-2 in human airway cells. Life Sci Alliance. 2020. https://doi.org/10.2650/lsa.202000786.
41. Dufour EK, Denault J-B, Bissonnette L, Hopkins PC, Lavigne P, Leduc R. The contribution of arginine residues within the P6-P1 region of $\alpha 1$-Antitrypsin to its reaction with furin. J Biol Chem. 2001;276(42):38971-9.

42. Jean F, Stella K, Thomas L, Liu G, Xiang Y, Reason AJ, et al. $\alpha 1$-Antitrypsin Portland, a bioengineered serpin highly selective for furin: application as an antipathogenic agent. Proc Natl Acad Sci. 1998;95(13):7293-8.

43. Jiao G-S, Cregar L, Wang J, Millis SZ, Tang C, O'Malley S, et al. Synthetic small molecule furin inhibitors derived from 2, 5-dideoxystreptamine. Proc Natl Acad Sci. 2006;103(52):19707-12.

44. Dahms SO, Jiao G-S, Than ME. Structural studies revealed active site distortions of human furin by a small molecule inhibitor. ACS Chem Biol. 2017;12(5):1211-6.

45. Dahms SO, Haider T, Klebe G, Steinmetzer T, Brandstetter H. OFF-state-specific inhibition of the proprotein convertase furin. ACS Chem Biol. 2021;16(9):1692-700.

46. Basak A, Banik UK, Basak S, Seidah NG, Li S (2006) Evaluation of anti-proprotein convertase activity of diterpene andrographolid derived products. In: Regulation of carcinogenesis, angiogenesis and metastasis by the proprotein convertases (PCs): Springer, Berlin, $\mathrm{p}$ 137-154

47. Lalou C, Basak A, Mishra P, Mohanta B, Banik R, Dinda B, et al Inhibition of tumor cells proliferation and migration by the flavonoid furin inhibitor isolated from Oroxylum indicum. Curr Med Chem. 2013;20(4):583-91.

48. Peng M, Watanabe S, Chan KWK, He Q, Zhao Y, Zhang Z, et al. Luteolin restricts dengue virus replication through inhibition of the proprotein convertase furin. Antiviral Res. 2017;143:176-85.

49. Haanen J, Carbonnel F, Robert C, Kerr K, Peters S, Larkin J, et al. Management of toxicities from immunotherapy: ESMO clinical practice guidelines for diagnosis, treatment and follow-up. Ann Oncol. 2017;28(suppl 4):119-42.

50. Janahi IA, Rehman A, Baloch NU-A (2018) Corticosteroids and their use in respiratory disorders. In: Ali Gamal Al-kaf (ed), p 47. https://doi.org/10.5772/intechopen.72147

51. Habtemariam S, Nabavi SF, Banach M, Berindan-Neagoe I, Sarkar $\mathrm{K}$, Sil PC, et al. Should we try SARS-CoV-2 helicase inhibitors for COVID-19 therapy? Arch Med Res. 2020;51(7):733-5.

52. Banach M, Penson PE, Fras Z, Vrablik M, Pella D, Reiner Ž, et al. Brief recommendations on the management of adult patients with familial hypercholesterolemia during the COVID-19 pandemic. Pharmacol Res. 2020;158:104891.

53. Katsiki N, Banach M, Mikhailidis DP. Lipid-lowering therapy and renin-angiotensin-aldosterone system inhibitors in the era of the COVID-19 pandemic. Arch Med Sci. 2020;16(2):485-9.

54. Reiner Ž, Hatamipour M, Banach M, Pirro M, Al-Rasadi K, Jamialahmadi T, et al. Statins and the Covid-19 main protease: in silico evidence on direct interaction. Arch Med Sci. 2020;16(2):490-6.

55. Zahedipour F, Hosseini SA, Sathyapalan T, Majeed M, Jamialahmadi T, Al-Rasadi K, et al. Potential effects of curcumin in the treatment of COVID-19 infection. Phytother Res. 2020;34(11):2911-20.

Publisher's Note Springer Nature remains neutral with regard to jurisdictional claims in published maps and institutional affiliations. 